\title{
On the Diophantine Equation $\sum X_{i}=\prod X_{i}$
}

\author{
By M. L. Brown
}

\begin{abstract}
The diophantine equation $X_{1}+\cdots+X_{k}=X_{1} \cdots X_{k}$ has at least one solution in positive integers for $k \geqslant 2$. The set of integers $k$ for which this is the only solution are investigated; in particular, this set is conjectured to be a known finite sequence.
\end{abstract}

The equation $f_{k}(\mathbf{X})=X_{1}+X_{2}+\cdots+X_{k}-X_{1} X_{2} \cdots X_{k}=0$ has the solution, for $k \geqslant 2$, given by $X_{1}=2, X_{2}=k, X_{3}=X_{4}=\cdots=X_{k}=1$. Schinzel showed that there are no other solutions in positive integers, apart from permutations of this given solution, for $k=6$ and $k=24$. Misiurewicz [2] states that $k=2,3,4,6,24$, $144,174,444$ are the only values of $k<1000$ for which $f_{k}(X)=0$ has essentially one solution, as above. But the number 144 in this list (given in both [2] and [1, D24]) is probably a misprint for 114 , for with this correction Misiurewicz's assertion is then correct (evidently 144 will not do because of the extra solution $1^{141} \cdot 2 \cdot 4 \cdot 21=168$ $=(141) \cdot 1+2+4+21)$. We report here on some further calculations with this equation.

Proposition 1. The equation $f_{k}(\mathbf{X})=0$, for $k \geqslant 4$, has only one solution in positive integers, apart from permutations, if and only if the following conditions hold:

(1) $k-1$ is a prime number.

(2) Let $s, n$ be any integers, if any, with $3 \leqslant s \leqslant \log _{2} k+1,2^{s-2} \leqslant n \leqslant$ $\left(k^{1 / s}+1\right)^{s-2}$ and $n$ being a product $x_{1} \cdots x_{s-2}$ of $s-2$ integers $x_{i} \geqslant 2$. Put $t=$ $x_{1}+\cdots+x_{s-2}$. Then no factor of $N=(k-s+t) n+1$ is congruent to -1 modulo $n$ except possibly for $n-1$ and $N / n-1$.

Proof. Let $f_{k}(\mathbf{x})=0$ be a solution in positive integers; we may suppose that $x_{1}, \ldots, x_{s}$ are precisely those integers among $x_{1}, \ldots, x_{k}$ which do not equal 1 . Thus $x_{1} \cdots x_{s}=k-s+x_{1}+\cdots+x_{s}$. Since $x_{i} \geqslant 2$ for all $i \leqslant s$, it follows that $2^{s} \leqslant k$ $+s$. It is then elementary to show that for $k \geqslant 4$ we have $s \leqslant \log _{2} k+1$.

The case $s=1$ is easily ruled out, so we next consider the case $s=2$. A solution $f_{k}(\mathbf{x})=0$ with $s=2$ gives $x_{1}\left(x_{2}-1\right)=k-2+x_{2}$. If this is distinct from the given solution we have that $x_{2}-1$ does not equal $k-1$ and is a proper factor of $k-2+x_{2}$. It follows that $x_{2}-1$ is a proper factor of $k-1$. Thus no other solution exists if and only if $k-1$ is a prime number.

Suppose now $s \geqslant 3$. Given integers $x_{1}, \ldots, x_{s-2} \geqslant 2$, put $n=x_{1} x_{2} \cdots x_{s-2}$ and $t=x_{1}+x_{2}+\cdots+x_{s-2}$. Then there are integers $x_{s-1}, x_{s} \geqslant 2$ with $f_{k}(\mathbf{x})=k-$ $s+t+x_{s-1}+x_{s-2}-n x_{s-1} x_{s-2}=0$ if and only if $f=x_{s-1} n-1$ is a factor of,

Received September 28, 1982.

1980 Mathematics Subject Classification. Primary 10B15.

Key words and phrases. Diophantine equation, prime numbers.

1984 American Mathematical Society $0025-5718 / 84 \$ 1.00+\$ .25$ per page 
and not equal to, $k-s+t+x_{s}$. Put $N=(k-s+t) n+1$. We deduce $x_{s}$, $x_{s} \geqslant 2$ exist as required if and only if $N$ has a factor $f \neq n-1$ and $\neq N /(n-1)$ with $f$ congruent to -1 modulo $n$. It remains to bound $n$.

Let $x_{1}, \ldots, x_{s}$ be a solution, as above, with the $x_{i}$ arranged in increasing magnitude. Consider the problem of maximizing $n$ subject to $f_{h}(\mathbf{x})=0, x_{i} \geqslant 2$, and the other stated constraints on $x_{i}$ except that we now allow nonintegral values. Since $n$, as a function of $x_{1}, \ldots, x_{s}$, has no critical values in the given region, it must take its extreme values for extreme values of the variables $x_{i}$. It is easy to see that for the maximum value of $n$ we must have all the $x_{1}, i \leqslant s$, being equal. Thus the maximum value of $n$ is $x^{s-2}$ where $x$ is the positive root of the equation $x^{s}=k-s+s x$. Plainly, $k^{1 / s} \leqslant x \leqslant k^{1 / s}+1$; thus $n \leqslant\left(k^{1 / s}+1\right)^{s \cdots 2}$ as required.

Corollary 1. Suppose $f_{h}(\mathbf{X})=0$ has only 1 essential solution in positive integers. Then:

(1) $k-1$ and $2 k-1$ are prime numbers.

(2) $6 \mid k$ if $k>4$.

(3) $4 k+1$ and $4 k+5$ are sums of two squares for $k>4$.

Proof. (1) Take $s=3, n=2$ in the proposition. (2) follows from (1). (3) Take $n=4, s=3$ or $n=2.2, s=4$ in the proposition and apply Fermat's criterion. noting from (2) that neither $4 k+1$ nor $4 k+5$ is divisible by 3 for $k>4$.

Proposition 1 can be used to give an algorithm for testing if an integer $k$ has the required property; the number of steps required is at most $O\left(k^{3 / 2+\varepsilon}\right)$, for all $\varepsilon>0$. Using this algorithm, a PET 4032 microprocessor was used to test suitable values of $k$; this revealed the discrepancy in Misiurewicz's list, though it is easy to check by hand using Proposition 1 that $k=114$ should be in the list. No other values of $k<11,000$ were found for which $f_{k}(\mathbf{X})=0$ has one solution. this computation taking 40 minutes of computing time. We thus end with:

ConjeCtURE. The only values of $k$ for which $f_{k}(\mathbf{X})=0$ has one solution are $k=2,3$. $4,6,24,114,174,444$.

Added in Proof. With a different program, the conjecture has now been verified for all $k \leqslant 50,000$.

Department of Pure Mathematics

University College

P.O. Box 78

Cardiff CFI IXL. Great Britain

1. R. K. Guy, Unsoleed Prohlems in Number Theon, Springer-Verlag. New York. Heidelberg. Berlin. 1981.

2. M. Misiurewicz., "Ungelöste Probleme,” Elem. Math., v. 21. 1966, p. 90. 